\title{
The performance of the Muon Veto of the GERDA experiment
}

\author{
K. Freund ${ }^{1,4}$, R. Falkenstein ${ }^{1}$, P. Grabmayr ${ }^{1, a}$, A. Hegai ${ }^{1}$, J. Jochum ${ }^{1}$, M. Knapp ${ }^{1,5}$, B. Lubsandorzhiev ${ }^{1,2}$, \\ F. Ritter $^{1,6}$, C. Schmitt ${ }^{1}$, A.-K. Schütz ${ }^{1}$, I. Jitnikov ${ }^{3}$, E. Shevchik ${ }^{3}$, M. Shirchenko ${ }^{3}$, D. Zinatulina ${ }^{3}$ \\ ${ }^{1}$ Physikalisches Institut, Eberhard Karls Universität Tübingen, Tübingen, Germany \\ ${ }^{2}$ Institute for Nuclear Research of the Russian Academy of Sciences, Moscow, Russia \\ ${ }^{3}$ Joint Institute for Nuclear Research, Dubna, Russia \\ ${ }^{4}$ Present address: maxment GmbH, Heidelberg, Germany \\ ${ }^{5}$ Present address: Areva, Rome, France \\ ${ }^{6}$ Present address: Bosch GmbH, Stuttgart, Germany
}

Received: 25 January 2016 / Accepted: 12 May 2016 / Published online: 25 May 2016

(C) The Author(s) 2016. This article is published with open access at Springerlink.com

\begin{abstract}
Low background experiments need a suppression of cosmogenically induced events. The GERDA experiment located at LNGS is searching for the $0 \nu \beta \beta$ decay of ${ }^{76} \mathrm{Ge}$. It is equipped with an active muon veto the main part of which is a water Cherenkov veto with 66 PMTs in the water tank surrounding the GERDA cryostat. With this system 806 live days have been recorded, 491 days were combined muon-germanium data. A muon detection efficiency of $\varepsilon_{\mu \mathrm{d}}=(99.935 \pm 0.015) \%$ was found in a Monte Carlo simulation for the muons depositing energy in the germanium detectors. By examining coincident muon-germanium events a rejection efficiency of $\varepsilon_{\mu r}=\left(99.2_{-0.4}^{+0.3}\right) \%$ was found. Without veto condition the muons by themselves would cause a background index of $\mathrm{BI}_{\mu}=(3.16 \pm 0.85) \times 10^{-3} \mathrm{cts} /(\mathrm{keV} \cdot \mathrm{kg} \cdot$ year $)$ at $Q_{\beta \beta}$.
\end{abstract}

\section{Introduction}

Muons may cause a substantial background to rare event searches like GERDA (Germanium Detector Array) by generating counts in the region of interest (ROI) either through direct energy deposition in the detectors or through e.g. decay radiation of spallation products. The GERDA experiment is searching for the neutrinoless double beta $(0 \nu \beta \beta)$ decay of ${ }^{76} \mathrm{Ge}[1,2]$. The experimental signature of the $0 \nu \beta \beta$ decay is a peak at $Q_{\beta \beta}$, the $Q$ value of the decay.

GERDA was constructed in the underground laboratory of Laboratori Nazionali del Gran Sasso (LNGS) of INFN in Italy, which offers an overburden of $3500 \mathrm{~m}$ water equivalent (m.w.e.) of rock and hence a reduction of the muon flux by a factor of $\sim 10^{6}$ to a rate of $\sim 3.4 \times 10^{-4} /\left(\mathrm{s} \cdot \mathrm{m}^{2}\right)$. This remaining muon flux, however, is sufficient to cause a non-negligible background in the region of interest around $Q_{\beta \beta}=2039 \mathrm{keV}$ when increasing the sensitivity beyond $T_{1 / 2}^{0 \nu}>10^{25}$ year or when requiring a background index $\mathrm{BI}<10^{-2} \mathrm{cts} /(\mathrm{keV} \cdot \mathrm{kg} \cdot$ year $)$. However, also other analyses profit from the reduced backgrounds (see e.g. Refs. [3, 4]).

The origin of muons at LNGS for Phase I of GERDA was twofold. First of all, the majority of the detectable muons are produced cosmogenically. Spectrum and angular distribution of the muons are both altered by the profile of the rock overburden and have been measured for LNGS with high precision [5]. These muons have an average energy of $\left\langle E_{\mu}\right\rangle=270 \mathrm{GeV}$. Second, a source for muons was the CNGS neutrino beam from CERN [6] which created muons via e.g. $v_{\mu}+d \rightarrow \mu^{-}+u$ reactions in the vicinity of the detector. This contribution amounted to $2.2 \%$ of the total muon flux in GERDA. As the CNGS was shut down after 2012 the future Phase II of GERDA will be unaffected. In order to reduce muon induced background, a muon veto comprised of a water Cherenkov veto and a scintillator veto was implemented to tag muons and to use its response as a veto signal in the $0 v \beta \beta$ analysis.

This paper describes the hardware and set-up of the veto and the DAQ system. The performance of the veto will be presented and compared to Monte Carlo simulations. Detection and rejection efficiencies for the veto will be discussed. Parts of this work have been published during the respective Ph.D. periods [7-11].

\footnotetext{
a e-mail: grabmayr@uni-tuebingen.de
} 


\section{Instrumentation}

Here, a technical description of the apparatus is given. Purpose and function of both parts of the veto, Cherenkov and scintillator panels, are introduced. The trigger logic, calibration, and data acquisition are summarized.

\subsection{GERDA}

In GERDA an array of bare germanium detectors is operated in a cryostat that contains $64 \mathrm{~m}^{3}$ of liquid argon (LAr) as seen in Fig. 1. The cryostat is surrounded by a water tank with a diameter of $10 \mathrm{~m}$ and a height of $9.4 \mathrm{~m}$ that is filled with $590 \mathrm{t}$ ultra-pure water; its walls are covered with a reflective foil. The cryostat has a connection (neck) through the water tank to the clean room above from which the germanium detectors are lowered into the LAr. Both water tank and cryostat are part of the innovative shielding design of GERDA where low-Z materials are used in order to reduce cosmogenic activation $[1,12]$.

The GERDA muon veto consists of two independent parts which are read out by the same DAQ. The first and main

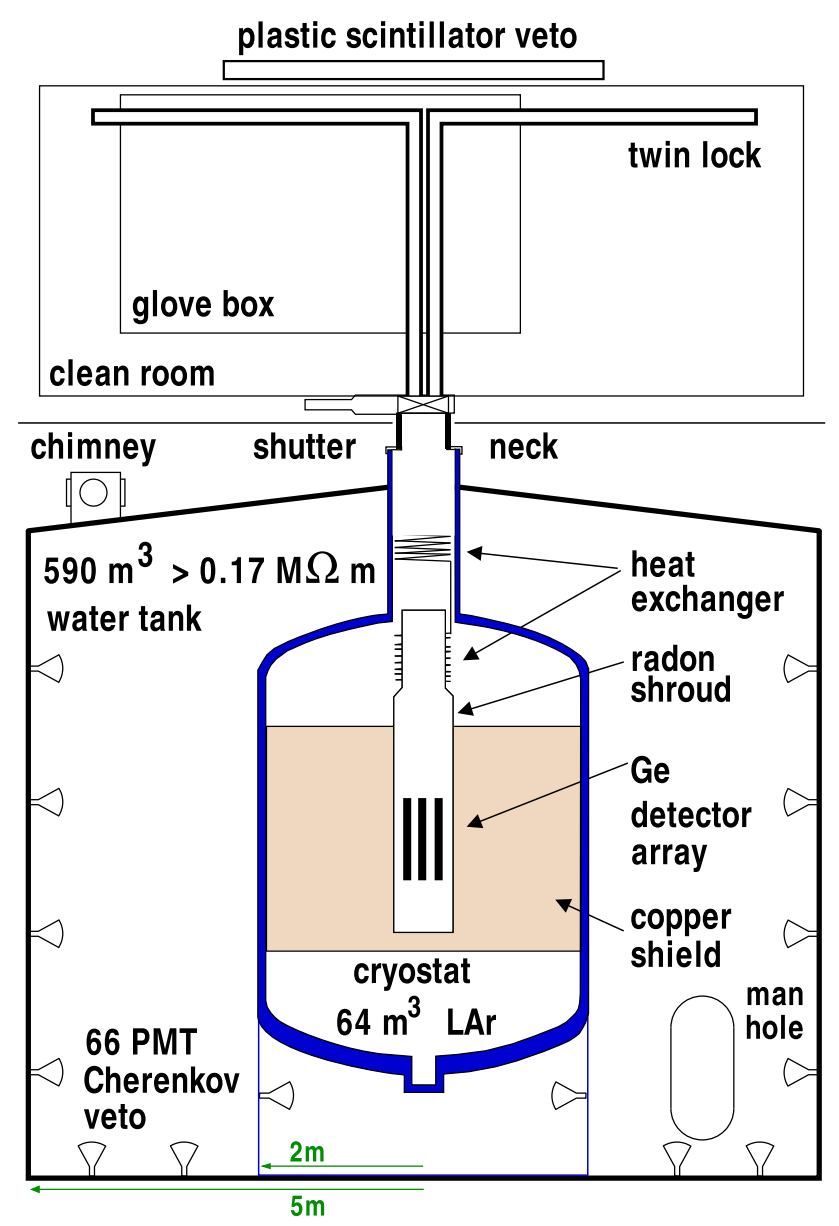

Fig. 1 A sketch of the GERDA experiment part is a water Cherenkov veto that detects the Cherenkov radiation of the traversing muons. Thus, the water tank is instrumented with 66 encapsulated photomultipliers (PMTs). The second part of the muon veto is comprised of plastic scintillator panels. Since the Cherenkov veto offers reduced tagging capability at or around the neck of the cryostat, plastic panels were placed on top of the clean room in order to close this weak spot in the Cherenkov veto.

\subsection{The Cherenkov veto}

Below the water level there are 66 PMTs ( 8 " size) of type $9354 \mathrm{~KB} / 9350 \mathrm{~KB}$ by ETL [13] installed. In order to protect the PMTs from the surrounding water, each PMT is housed in a stainless-steel encapsulation which is further developed from the design of the BOREXINO capsules [14]. The capsule of low radioactivity stainless steel [15] is closed with a custom made PET cap and is filled with IR spectroscopy oil [16]. The oil keeps the optical transition between PET cap and PMT as smooth as possible. Thus, efficiency losses by total internal reflections are minimized. The bottom of the base of the PMT is encased in polyurethane [17] and sealed with silicon gel [18]. The lower part of the PMT, i.e. the dynode structure, is protected from magnetic fields by a cone of $\mu$-metal [19]. Each capsule is equipped with an optical fiber for optical calibration pulses from outside the water tank. The new features comprise the individual fibers and the direct connection of the high-voltage cable with the base avoiding an additional water-proof connector. To keep the number of cables and connectors within the clean water at a minimum an underwater high-voltage cable [20] connects the PMT to a signal splitter outside of the water tank. This band-pass separates the signal from the high voltage (HV). The former is digitized and stored on disk upon a trigger (Sect. 2.4). The HV is supplied by a HV multichannel system by CAEN [21] equipped with six 12-channel boards [22].

A sketch of the capsule is shown in Fig. 2 and images of the components of the Cherenkov veto are shown in Fig. 3.

The first water filling of the GERDA water tank was provided by the BOREXINO water plant. Thereafter, the water is recirculated and purified (see Sect. 4.2 of Ref. [1]). The purification system which consists of filters, de-ionizers, and an osmosis unit is running with $2.4 \mathrm{~m}^{3} / \mathrm{h}$ and keeps the water at the ultra-pure level of $>0.17 \mathrm{M} \Omega \mathrm{m}$ [23].

The PMTs are arranged in seven rings in the water tank, their distribution is shown in Fig. 4. One ring of six PMTs (101-106) is pointing inside the separate volume under the cryostat (commonly referred to as "pillbox"), two rings of eight PMTs (201-208) and 12 PMTs (301-312), respectively, are placed on the bottom of the water tank looking upwards and four rings of 10 PMTs are placed on the wall (401710 ), pointing horizontally toward the cryostat. The number 


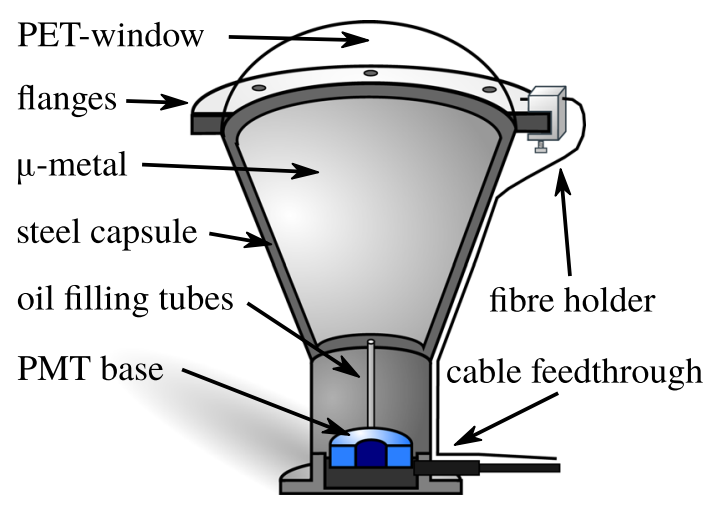

Fig. 2 Parts of a PMT capsule

of PMTs and their placement was chosen after an extensive Monte Carlo study [7]. The PMTs closest to the cryostat, i.e. those from the pillbox and the inner ring on the bottom, were selected according to their performance and radioactivity (type $9354 \mathrm{~KB}$ is a low activity module). However, due to the distance to the germanium detectors and the low overall mass the radioactivity of the muon veto is negligible compared to the one of the stainless steel cryostat [7].

The floor and the walls of the water tank and of the cryostat are covered with the reflective Daylighting Film DF2000MA (commonly known as "VM2000") [24] which offers a reflec-

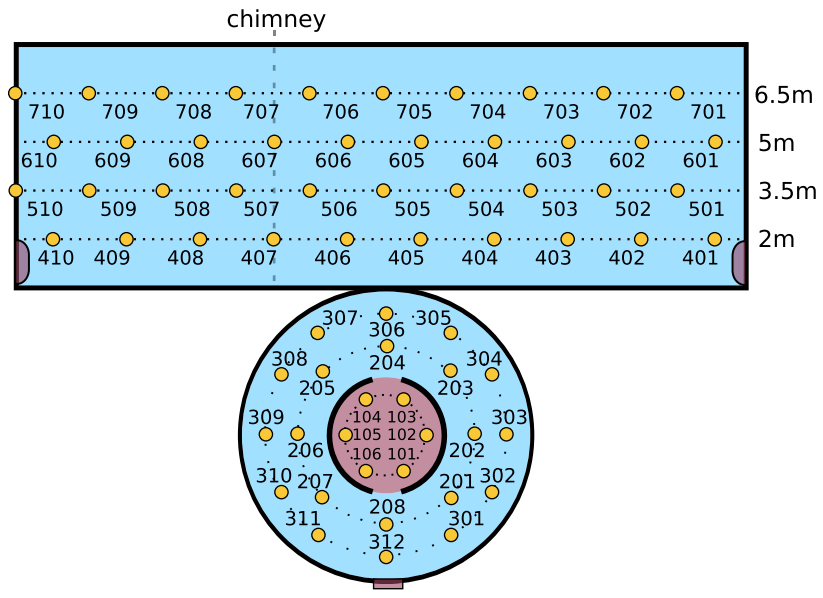

Fig. 4 A sketch of the PMT distribution inside the GERDA water tank. The violet area on the bottom plate signifies the circumference of the cryostat and the gaps show the position of manholes into the pillbox. The other three violet areas show the location of the manhole into the cryostat. All cables and fibers are lead out of the water tank through three $50 \mathrm{~cm}$ flanges mounted in a "chimney" above the water level (Fig. 1)

tivity of $>99 \%$ in order to increase the light yield of each event. This comes at the cost of a reduction in tracking capabilities of each individual muon. The reflective foil does not only increase the collected light yield of each muon, but it acts
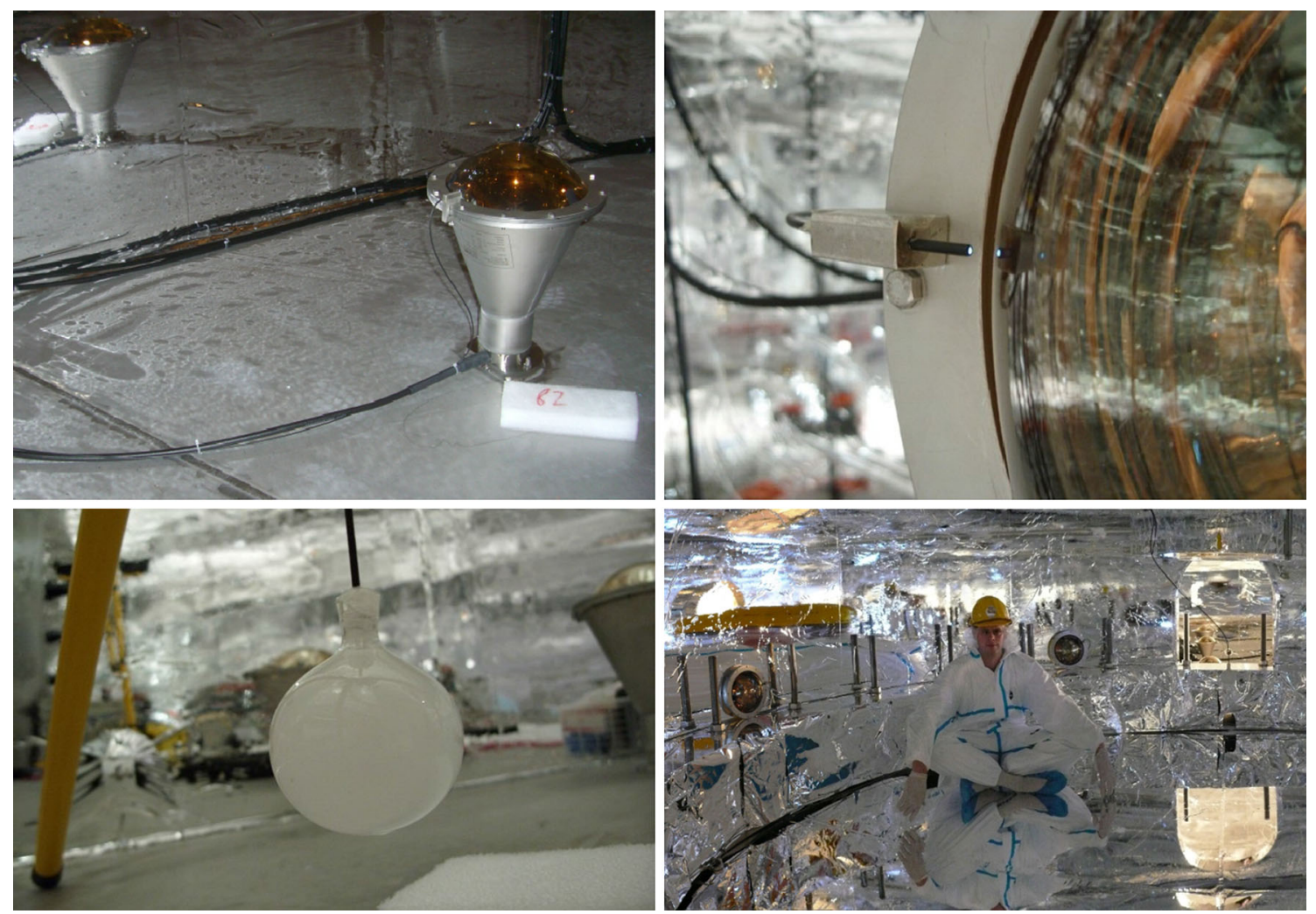

Fig. 3 Images of the components of the Cherenkov veto during the installation. Top, left a capsule mounted on the floor; top, right a capsule on the wall; bottom, left a diffuser ball; bottom, right PMTs and Daylighting Film in "pillbox" below the cryostat 


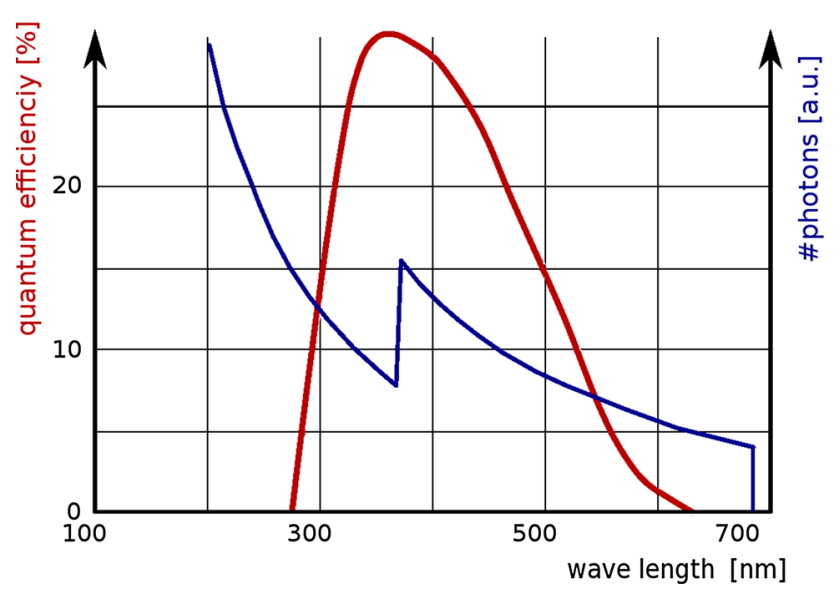

Fig. 5 Efficiency (red curve) of a 9354KB type PMT adapted from Ref. [13] and simulated photon spectrum with wavelength shifting effect (blue histogram)

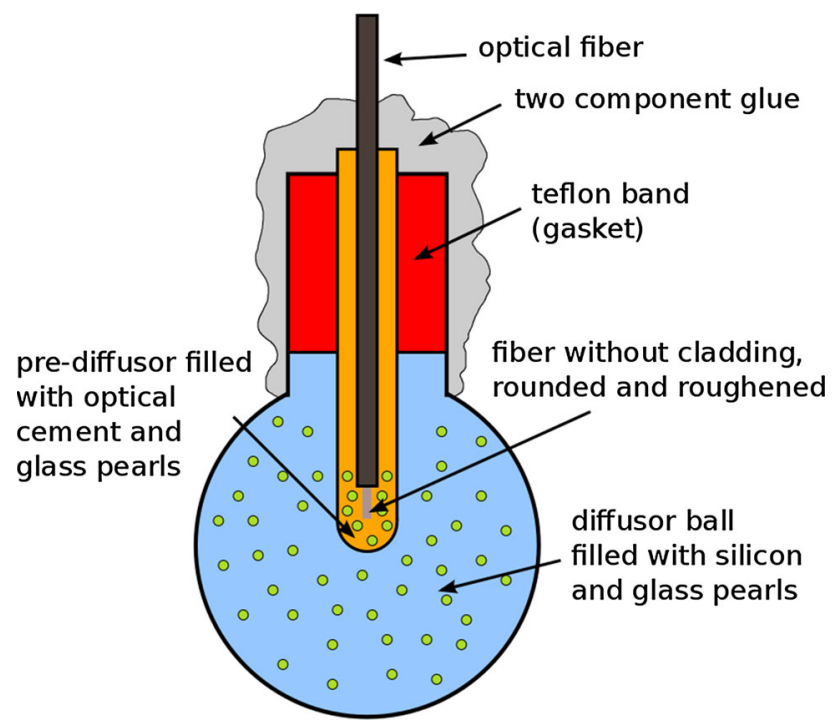

Fig. 6 Schematic drawing of a diffuser ball after Ref. [11]

as a wavelength shifter as well. It shifts the predominantly ultraviolet Cherenkov photons to around $400 \mathrm{~nm}$ where the PMTs are most efficient. The efficiency of a 9354KB type PMT and the wavelength shifting effect implemented in the GEANT4 simulations are shown in Fig. 5. A radio assay has been performed with the spectrometer GEMPI [25] that resulted in the following values and limits with a confidence level of $95 \%$. The specific activities are given in units of $\mathrm{mBq} / \mathrm{kg}$ by $<1.6$ for ${ }^{226} \mathrm{Ra},<2.2$ for ${ }^{228} \mathrm{Ra},<1.2$ for ${ }^{228} \mathrm{Th}$, $140 \pm 10$ for ${ }^{40} \mathrm{~K},<0.44$ for ${ }^{60} \mathrm{Co}$, and $<0.45$ for ${ }^{137} \mathrm{Cs}$ [26].

The PMTs are calibrated regularly with a set of five custom made diffuser balls shown in Fig. 6 which are constructed to provide a light source as isotropic as possible. These are glass balls with a diameter of $4.5 \mathrm{~cm}$ that are filled with a mixture of silicon gel [18] and glass pearls [27] with a diameter of $\sim 50 \mu \mathrm{m}$. An optical fiber is glued into a small vial inside the ball with a higher content of glass pearls. The cladding at the end of the fiber is removed and the fiber is roughened in order to obtain a diffuse light emission. Ultra-fast LEDs outside of the water tank can be pulsed to illuminate the five diffuser balls [11]. Four of these balls are distributed in the main water tank and a fifth is placed inside the "pillbox". With an appropriate setting of the LEDs it is possible to illuminate all PMTs simultaneously with single photons and thus record single-photon responses of every PMT at the same time. As the diffuser balls are working very well, the calibration fibers attached to every PMT are currently not in use.

\subsection{Scintillator Veto}

Muons passing through the neck of the cryostat may either traverse a too short distance in the "pillbox" or in the water tank to be detected. In order to keep the muon rejection efficiency as high as possible, a veto of plastic scintillator panels was conceived and installed.

Each scintillator panel contains a $200 \times 50 \times 3 \mathrm{~cm}^{3}$ sheet of plastic scintillator based on polystyrol with an addition of PTP $(2 \%)$ and POPOP $(0.03 \%)$ [28]. In addition the panels contain optical fibers [29] on the narrow sides as lightguides, an electronics board with a trigger and shaper and a PMT. Half of the panels are equipped with PMTs by Hamamatsu Photonics [30] and the other half with PMT-085 by Kvadrotech. The PMT-085 are powered by the same HV supply as the PMTs of the Cherenkov part of the muon veto, however, connecting 3 PMTs to one HV output. The front-end electronics, the Hamamatsu PMTs and all other components are powered by a custom made power source with $+12 \mathrm{~V}$ and $\pm 6 \mathrm{~V}$.

The panels are arranged in three layers covering an area of $4 \times 3 \mathrm{~m}^{2}$ centered over the neck of the cryostat. It was aimed to keep both trigger rate and thus data volume of the veto as low as possible. Therefore, the trigger is a triple coincidence of all three layers since this option offers a high discrimination against non-muonic background events in the panels such as $\gamma$ rays from environmental radioactivity. Thus, the scintillator veto records an almost pure muon sample. Individually, each panel shows a pulse-height distribution which takes the form of a Landau peak. Despite the triple coincidence, small $\gamma$ contamination at low pulse heights remains. These events can be discarded with a product cut of the form

$\left(x_{1}-\rho_{1}\right)\left(x_{2}-\rho_{2}\right)<c$

for the pulse heights $x_{1,2}$ of any pair of panels in the stack and constants $\rho_{1,2}$ and $c$. The 2-D pulse-height distribution of two panels in a stack, the applied cut and a spectrum of a single panel with a fit to the Landau peak is shown in Fig. 7. 


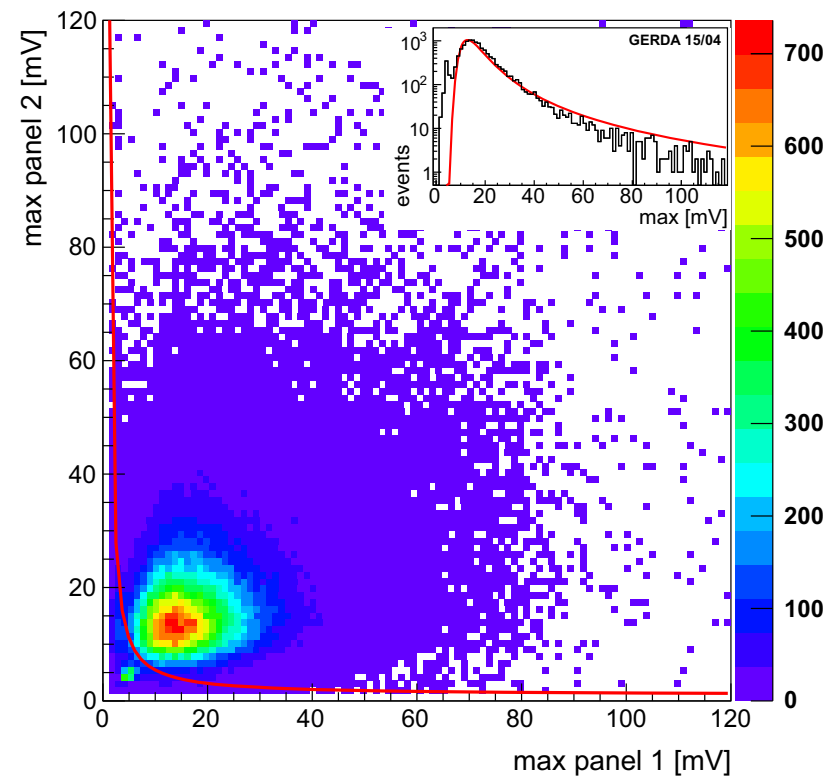

Fig. 7 Scatter plot of the pulse-height maxima of two panels in a triple stack with aproduct cut (red line) as given in Eq. 1. The inset shows the pulse-height distribution of one panel and a Landau-fit (red)

\subsection{Data acquisition}

The entire apparatus is read out and operated by a VME data acquisition system (DAQ) which is almost identical to the one of the germanium DAQ (see Ref. [1] for details). Ten Flash-ADCs [31] with 8 channels each digitize the input signals of the Cherenkov veto with $100 \mathrm{MHz}$. The signals in each channel are processed by a trapezoidal filter and if the height exceeds the threshold set to 0.5 photo-electrons (p.e.) an internal trigger is generated. Each FADC module has one trigger output which is the logic OR of the internal triggers of its eight channels. Thus, the PMT signals are connected to the input channels in such a way over the FADCs that neighboring PMTs are always read out by different FADCs. This allows the proper detection of clustered events in which PMTs next to each other have fired. The final trigger condition is set such that five FADCs must trigger on at least 0.5 photo-electrons (p.e.) each within $60 \mathrm{~ns}$. That signal is realized by a FPGA and it starts the readout of all traces covering a period of $4 \mu \mathrm{s}$. This time setting was the minimum range compatible with the DAQ for the germanium data stream. Furthermore, it amply covered all the light from a muon which might be stretched over a few $100 \mathrm{~ns}$ due to the high reflectivity of the VM2000 foil. The traces are stored together with the time stamp, during Phase I from a GPS clock. A schematic drawing of the entire muon veto and its data flow is shown in Fig. 8. The trigger signal is furthermore sent to the germanium DAQ recording it as a redundant but immediate veto information for the germanium data stream.

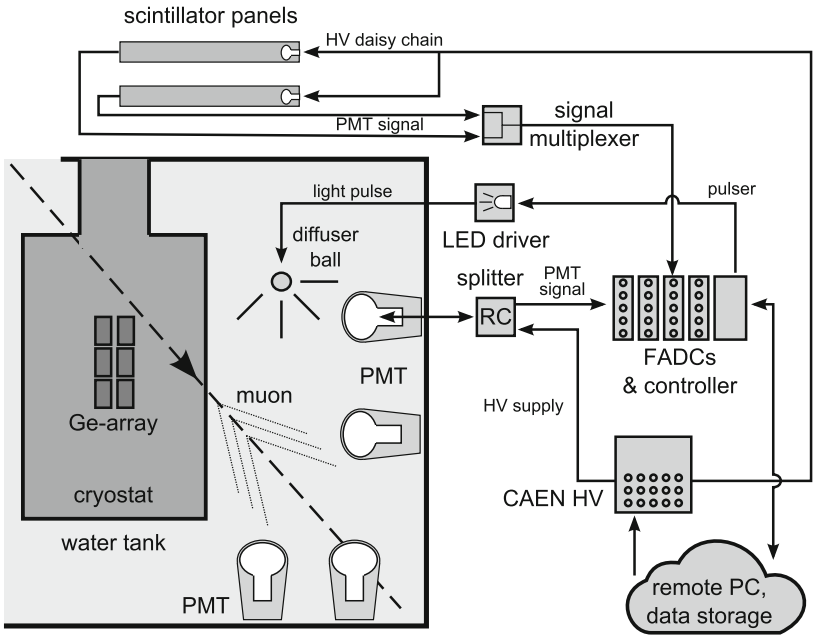

Fig. 8 Schematic drawing of the data acquisition (DAQ) set-up

The scintillator panels are arranged in three layers of 12 panels each. Three additional FADCs of the same type digitize the signal of the scintillator veto. The signals of two non-neighboring panels within a layer are multiplexed onto one FADC channel using custom made reflection-free modules with an amplification of $-6 \mathrm{~dB}$. Thus the 36 panels occupy only 18 FADC channels such that each layer is read out by one FADC module. The panel stack which was hit can be determined by the unique combination of fired channels. The same trigger logic as for the Cherenkov veto is applied for the panels, albeit the trigger window is larger in order to accommodate the much longer output signals of the panel PMTs because they are shaped with a larger time constant. For a panel event, all three FADCs (i.e. all three panels in a stack) need to have fired. Both types of trigger signals are accepted during data taking.

For a calibration run of the PMTs, the standard data taking is stopped. The ultra-fast calibration LEDs are activated with a pulser and the LED luminosity is controlled by a current source in form of a digital-to-analog converter [32]. A separate FADC reads out the pulser signal and triggers the entire veto for each pulse.

Two calibration spectra can be seen in Fig. 9. One is showing the conventionally recorded single-photon peak (SPP) set to channel 100, the other comes from a too bright LED setting. This causes a contamination of the SPP by the double photon peak (DPP) and a shift of the amplitudes of both peaks to higher values. The SPP emerges very well in most PMTs and peak-to-valley ratios between 1.2 and 3.0 are observed.

\section{Veto performance}

The GERDA muon veto was installed in 2009 and its operation started in November 2010. During the germanium commis- 


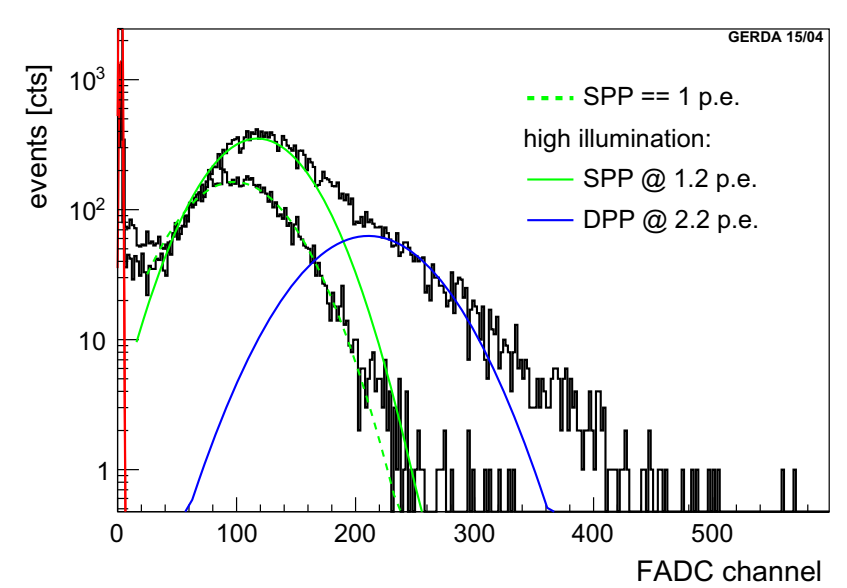

Fig. 9 Cherenkov PMT response with different forward voltages of the calibration LEDs, i.e. luminosities. With a low luminosity, only the single-photon peak (SPP, broken green line) is visible, if the luminosity is too high, the double photon peak (DPP, blue) emerges as well. The pedestal is shown in red

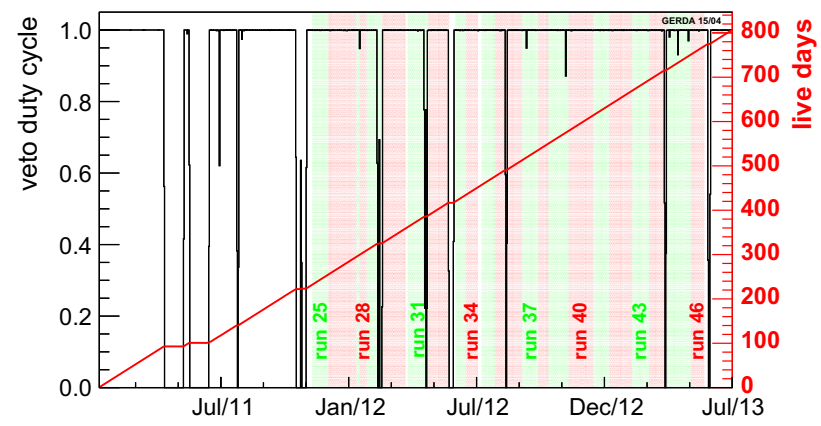

Fig. 10 Duty cycle of the muon veto. The veto uptime (black) and accumulated live days (red) are indicated as well as the GERDA physics runs for Phase I (filled light red and green)

sioning runs the panel veto was installed so that the complete veto was operational at the start of the physics runs of GERDA in November 2011. Until July 2013, 805.6 live days have been recorded and 491 days of combined muon-germanium data. The duty cycle is shown in Fig. 10 together with the accumulated live time (red line). During Phase I the muon DAQ was only stopped during breaks of the germanium data taking in order to perform short maintenance work and to calibrate the PMTs by adjusting the HV of each PMT so that each module shows the same response to single photons. The PMTs were very stable and since the beginning only few PMTs needed to be readjusted. As example, the rate per day is shown for selected seven PMTs in Fig. 11. The offsets are for display only. For e.g. PMT101 there was no readjustment necessary since May 2011, while PMT301 needed several tunings of the HV. However, between the breaks the light output remains stable. During the germanium data taking the muon veto was always fully operational.

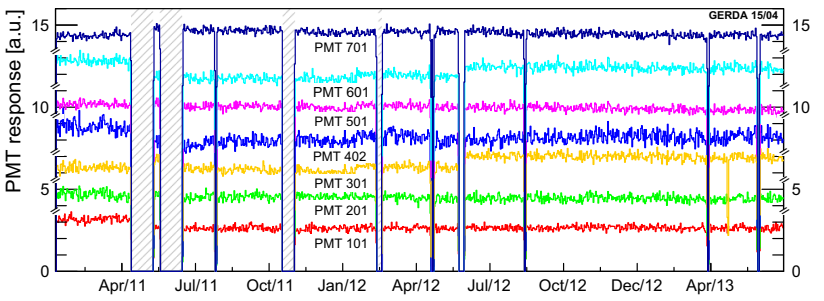

Fig. 11 Summary of the ratestability for selected PMTs; note the arbitrary offsets. The hatched areas indicate maintenance periods

During Phase I two PMTs were lost due to implosion of the tube (PMTs 401 and 604). The implosion was mostly contained by the encapsulation and no other PMTs in the vicinity were harmed. The implosions happened in February and April 2012 and the PMTs were over $10 \mathrm{~m}$ apart. Hence a direct influence can be ruled out. A third PMT was lost right after installation due to a punctured cable (PMT 305) and a fourth PMT ceased working during the installation and could be immediately exchanged (PMT 203). In July 2013, the GERDA water tank was drained, the veto inspected and two of these PMTs were successfully exchanged (PMTs 305 and 401). All other PMTs still work as intended and showed little to no signs of deterioration.

\subsection{Simulation studies}

The performance of the muon veto was simulated with the GEANT4-based [33] framework for GERDA and MAJORANA (MAGE) [34]. First, the simulations were used to find initial placements and efficiencies [7] and repeated once the exact geometries of the apparatus were finalized [9]. The muon spectra provided by the MACRO experiment were used as input for the simulation of cosmogenic muons [5]. The simulations were mainly used to determine the efficiency of the apparatus in the case the muon caused any energy deposition in the germanium crystals.

For the efficiency of the Cherenkov veto the simulation was undertaken in two parts. First, muons were simulated with the Cherenkov effect switched off. The primary vertices of those muons that caused energy deposition in the germanium detectors were extracted from these events. Second, these selected muons were used in a second simulation with Cherenkov effect enabled. This two-step procedure was applied in order to accelerate the simulation as only a minute fraction of the muons interact with the germanium detectors and because the simulation of optical photons is a resource-demanding procedure. A detection efficiency for the veto was derived by determining the fraction of energydepositing muons which caused a trigger signal in the muon veto. The trigger condition were the same as for the muon veto DAQ described in Sect. 2.4. For the entire veto a detection efficiency of 
$\varepsilon_{\mu d}^{\mathrm{MC}}=(99.935 \pm 0.015) \%$

for muons with energy deposition in the germanium detectors was found in the simulated data.

By removing certain PMTs from the efficiency calculations, a veto degradation (e.g. possibly broken PMTs or malfunctioning FADCs) was simulated. Even with the first two FADCs removed (14 PMTs in total, two in each of the seven rings shown in Fig. 4), the efficiency is still $\left(99.525_{-0.035}^{+0.025}\right) \%$. However, when only four PMTs in the pillbox are removed the value drops to $(97.855 \pm 0.065) \%$. The pillbox PMTs can hence be considered the most critical ones in the case of a break-down.

The light produced inside the pillbox can illuminate the main water tank through two small manholes. However, the light coming from these two holes is not sufficient in order to generate a trigger. The insensitivity against a loss of a few PMTs gives high reliability of the efficiency of the veto against small variations of trigger conditions or changes in the amplitude of the PMTs.

In an earlier work the efficiency was estimated as $\varepsilon_{\mu d}^{\mathrm{MC}}=$ $(99.56 \pm 0.42) \%$ and thus some what lower despite lower trigger conditions [7]. The previous simulation neglected the optical connection between the "pillbox" volume and the main water tank completely. Several other simulation studies have been performed like the veto response to regular cosmogenic muons which are shown and compared to experimental data in the next sections.

\subsection{Multiplicity and photon spectra}

The light yield of a single muon is determined by observing the total number of recorded p.e. in all PMTs. For comparison, the pillbox is treated as an individual volume. In Fig. 12 histograms of the recorded and simulated events are shown for the period of Phase I. In the spectra for the cosmogenic muons, apart from the very low light yield there are maxima at about 167 p.e. in the pillbox data and about 605 p.e. for the total spectrum. These broad peaks correspond to the mean traversed distance of $1.8 \mathrm{~m}$ for the pillbox and of $9 \mathrm{~m}$ for the water tank for muons with a mean incident angle of $60^{\circ}$. Light from muons in the water tank is subject to attenuation, the attenuation length of photons in water being $\sim 10 \mathrm{~m}$. Assuming a mean distance of $5 \mathrm{~m}$ of the PMTs from the muon track in the water tank and $2 \mathrm{~m}$ in the pillbox, muons of any track deposit approximately the same amount of light. Thus, each muon generates $(115 \pm 39)$ p.e./m. This is reproduced by the simulation.

A peak structure is visible in the data for muons which deposit energy in the germanium detectors as well. The peak for the pillbox is at slightly lower p.e. values because the average incident zenith angle is lower and thus the track length shorter. The p.e. spectrum for all PMTs shows a double peak

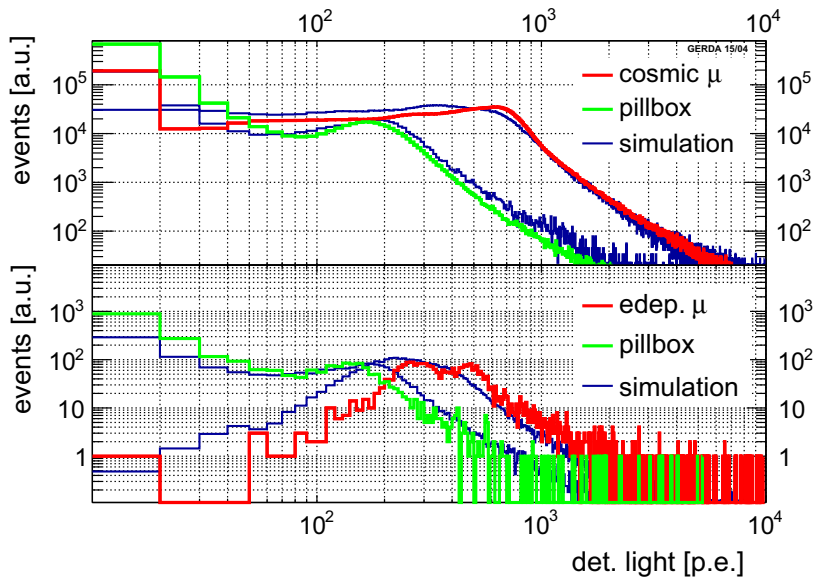

Fig. 12 Photo-electron spectra for all cosmogenic muons (top) and those with energy deposition in the germanium detectors (bottom). In each panel the total recorded p.e. spectrum (red) is compared to the spectrum which is recorded just in the pillbox (green). Spectra derived from simulations (blue) are normalized to the same exposure

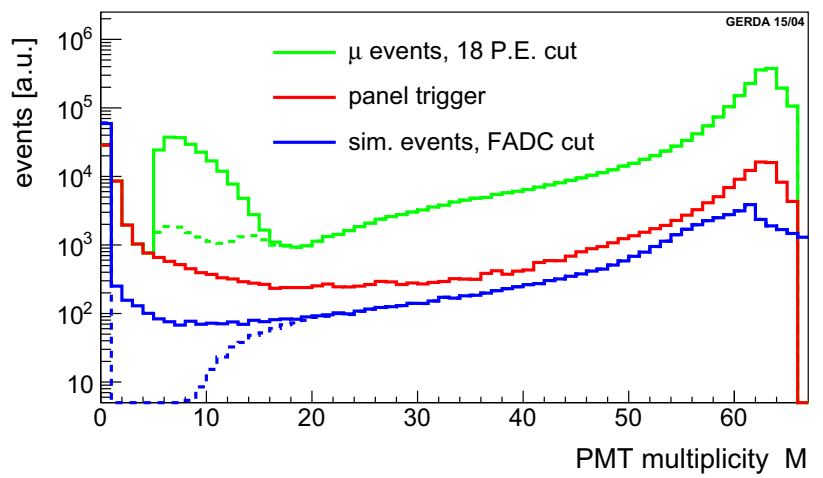

Fig. 13 PMT multiplicity spectrum of the Cherenkov veto. The multiplicity of all events (green) is compared with events where the panels have fired as well (red) and with simulated data (blue). Histograms after cuts are marked by dashed lines

feature. This is due to the fact that the muon has to cross the cryostat in order to deposit energy in the germanium. The muon can deposit light in the water tank before and after interacting with the germanium detectors. The higher peak corresponds to muons which pass the tank twice and the smaller corresponds to muons that pass the water tank just once (e.g. shallow angles close to the neck of the cryostat). Again, the simulations agree with this even though the double peak structure is less pronounced.

Another characteristic of a muon event is the number of fired PMTs. This multiplicity $M$ is shown in Fig. 13 for several classes of muon events. The spectrum of all measured events (green line) shows a peak at $M>60$ and another one at $M<10$. The peak at high $M$ is the regular response of the veto to muons. This is verified by either the simulated data (blue line) and a subset of all events in which the panel veto has to have fired as well (red line). The shape of the spectra 
is slightly different which is due to different incident angles (panel trigger) or the lack of random coincidences at low multiplicities in the simulated data. In addition, four PMTs were lost in the Cherenkov veto which enhances the peak at $M>60$. This is due to events that would trigger all PMTs are now recorded as having an $M=(66-x)$, where $x$ is the amount of lost PMTs. Thus, the counts at or just below 66 are shifted to lower multiplicities.

Only the measured spectrum from the Cherenkov trigger shows the low multiplicity enhancement, which is characterized by not only a low number of fired PMTs but also an unusually low amount of recorded p.e. With a cut of 18 p.e. this enhancement can be almost entirely suppressed. This is the standard cut condition for the Cherenkov veto data. The source of this enhancement is discussed in Sect. 3.7. A cut which emulates the trigger condition implemented in the DAQ described in Sect. 2.4 ("FADC cut") was applied to the simulated data. The resulting spectrum (dashed blue line) indicates the behavior of the Cherenkov veto without unphysical events at low $M$ like random coincidences or the low multiplicity enhancement.

\subsection{Coincident muon-germanium events}

The muon veto and the germanium systems have been operational in common during Phase I of GERDA over a time of 491 days. During this period an exposure of $\mathcal{E}=\left(21.6_{\mathrm{enr}}+\right.$ $6.2_{\text {nat }}$ ) $\mathrm{kg} \cdot$ year of germanium data was recorded. During Phase I the muon veto was only shut down during pauses in the germanium data taking and hence there is no additional loss of exposure due to the veto.

The two data streams were correlated by using the timestamps of the events. Prior to Phase I both DAQ systems were operated with their own internal clock which permitted undesired jumps in the time offset between the two systems. For Phase I both DAQ systems were equipped with the same GPS timing system so that events can be correlated via the timestamp with high precision. The length of the germanium trace of $160 \mu \mathrm{s}$ is taken as a coincidence window. Most interactions between muons and the germanium array happen within $\pm 10 \mu \mathrm{s}$, however, delayed interactions cannot be excluded. The germanium DAQ is described in detail in Ref. [1].

Both systems were physically and electronically very stable over time. After the deployment of the new BEGe detectors in the second half of Phase I the set-up of operational modules was unchanged for the rest of the data taking with four BEGes, six ${ }^{\text {enr }} \mathrm{Ge}$ and one ${ }^{\text {nat }} \mathrm{Ge}$ coaxial detector. In this period the mean rate was $r_{\mu}=(4.01 \pm 0.04) \times 10^{-2} / \mathrm{s}$ for the veto (no cuts applied), $r_{\mathrm{Ge}}=(2.87 \pm 0.06) \times 10^{-2} / \mathrm{s}$ for all germanium detectors and $r_{\text {coin }}=(9.5 \pm 0.6) \times 10^{-5} / \mathrm{s}$ the rate of physical coincidences. Due to the low rate of veto and germanium random coincidences are negligible compared to the true coincident rate.

\subsection{Muon rejection efficiency}

A muon rejection efficiency (MRE) can be obtained by defining a cut for clearly identified muon hits in the germanium detectors and testing for coincident veto signals. The rejection efficiency $\varepsilon_{\mu r}$ is given as the ratio of these events which are vetoed in comparison to the entire set. The following cuts were applied to the germanium events of the GERDA Phase I data to identify muons: either a single hit showed an energy depostion of more than $8.5 \mathrm{Mev}$ or the summed energy of a multi-hit exceeded $4 \mathrm{MeV}$. This cut excluded energy depositions from the $\mathrm{U}$ and Th decay chains. In addition, the germanium test-pulse and quality cuts were applied, but no muon veto cuts. Out of the 848 candidate muon events identified according to energy release and multiplicity in the Germanium detectors, 841 are actually in coincidence with a valid muon veto signal. This leads to a MRE of

$\varepsilon_{\mu r}=\left(99.2_{-0.4}^{+0.3}\right) \%$

This is a conservative number since the studied events are not standard events either in germanium multiplicity or in an energy range where saturation might set in.

The derived MRE is slightly lower in comparison to the efficiency derived from the simulation given in Eq. 2. Assuming that the given MRE can be projected to standard events, i.e. with multiplicity $m=1$ and with energies at or around the ROI, the rejection power of the veto is reliably high and close to unity.

\subsection{Muonic background index}

In order to estimate the improvement of the background index given in cts $/(\mathrm{keV} \cdot \mathrm{kg} \cdot \mathrm{year})$ due to the muon veto, $\mathrm{a} \pm 100 \mathrm{keV}$ window was chosen around $Q_{\beta \beta}$. Analog to the germanium background a blinding window of $\pm 20 \mathrm{keV}$ around $Q_{\beta \beta}$ was omitted from the analysis hence the ROI of this study is $160 \mathrm{keV}$ wide. Out of a total exposure of $\mathcal{E}=27.8 \mathrm{~kg} \cdot$ year of germanium data, 14 vetoed events were found in this ROI with a germanium multiplicity of one. Were these 14 events not vetoed, they would have led to a contribution to the BI of:

$\mathrm{BI}_{\mu}=(3.16 \pm 0.85) \times 10^{-3} \mathrm{cts} /(\mathrm{keV} \cdot \mathrm{kg} \cdot$ year $)$

Out of these 14 events 13 were accompanied with a veto response of 55 or more Cherenkov PMTs and the 14th exhibited a clear signal having fired 28 PMTs. All events produced the veto trigger up to $250 \mathrm{~ns}$ prior to the germanium trigger that was homogeneously distributed over all the germanium detectors. Due to the low statistics no pattern for these coincident events could be identified. A simulated value for the 
muonic background in the germanium array surviving anticoincidence cuts is [35]

$\mathrm{BI}_{\mu}(\mathrm{MC})=(1.6 \pm 0.1) \times 10^{-3} \mathrm{cts} /(\mathrm{keV} \cdot \mathrm{kg} \cdot$ year $)$.

As this simulation was undertaken before construction of the experiment was finalized, the geometry differs from what was realized. Due to the different geometry, the low statistics and the subsequent large errors, both results cannot easily be compared but are within the same order of magnitude.

With $\mathrm{BI}_{\mu}$ and the previously derived MRE, an estimation of the unvetoed background contribution can be given. It is assumed that the MRE is constant over the entire energy range of the germanium detectors. The given vetoed $\mathrm{BI}$ is equivalent to the amount of successfully vetoed muons, i.e. $99.2 \%$. An amount of unvetoed muons is found of

$$
\mathrm{BI}_{\mu, \text { unveto }}=(2.87 \pm 0.77) \times 10^{-5} \mathrm{cts} /(\mathrm{keV} \cdot \mathrm{kg} \cdot \text { year }) .
$$

The design goal of Phase II of GERDA aims to reach a total $\mathrm{BI}$ of $10^{-3} \mathrm{cts} /(\mathrm{keV} \cdot \mathrm{kg} \cdot$ year $)$. Thus, with the current settings of the muon veto unchanged, unvetoed muons would contribute $1 / 40$ of the BI "allowance". For Phase I of GERDA this is equivalent to 0.16 events in a $200 \mathrm{keV}$ analysis window.

\subsection{Panel detection efficiency}

In order to determine the efficiency of the panels, a data sample of clearly identified muons was selected. A cut on the Cherenkov events of $M \geq 20$ was chosen which discards unphysical events at low $M$. The pre-selection of muon events by the Cherenkov veto is necessary because the standard trigger of the plastic veto cannot be used. For the panels a simple cut on the pulse heights is insufficient due to the $\gamma$ coincidences at low pulse heights (see Fig. 7). In addition to the pulse-height cut defined by the trigger threshold, a cut on the product of two pulse heights of the form of Eq. 1 was applied. The detection efficiency of one panel can now be given as the ratio of events, in which the top and bottom panel of a stack have fired in comparison to the events where all three panels in a stack have fired.

The data set contained events since the beginning of the muon data taking with the panels in August 2011. In this set, 30,044 events were found which triggered the top and bottom panel. Of these events 29,951 triggered the third panel as well. This leads to an average muon detection efficiency per panel of:

$\varepsilon_{\mu d}^{\mathrm{P}}=\left(99.70_{-0.05}^{+0.03}\right) \%$

Since this value is an average over different panels, it can be seen as an approximation for a general panel efficiency. The efficiency of a triple stack of panels is hence $\left(\varepsilon_{\mu d}^{\mathrm{P}}\right)^{3}=$ $\left(99.10_{-0.15}^{+0.09}\right) \%$. Due to insensitive areas at the panel borders (scintillator edges, encasing) the effective area of the panels is reduced by $\sim 5 \mathrm{~mm}$ per border or less than $<0.25 \%$ of the area.

\subsection{Low multiplicity enhancement}

The enhancement at low multiplicities is characterized by a very low number of recorded photo-electrons (in most cases one or two p.e. per PMT) without any observable clustering effects and amounts to about $8.7 \%$ of the overall Cherenkov activity (i.e. $3.1 \times 10^{-3} / \mathrm{s}$ ). This behavior suggests a very faint source of light inside the water tank that is not directly caused by muons. Events which trigger the panel veto and can hence be considered true muon events do not show this anomaly. It was already suggested that this anomaly is caused by scintillation of the reflective foil under irradiation by $\alpha$ sources in the stainless steel of the water tank $[1,8]$. The foil has a highly reflective front side and is covered with an adhesive on the back side. According to the manufacturer the foil itself has a thickness of $66 \mu \mathrm{m}$ and the adhesive a thickness of $38 \mu \mathrm{m}$ [24]. If the adhesive can be assumed to be an organic compound a mean free path of $\sim 30 \mu \mathrm{m}$ for a $5 \mathrm{MeV} \alpha$ particle is expected. Hence it is unlikely that $\alpha$ particles coming from the back side are able to deposit energy in the foil and produce scintillation photons which can exit the foil on the front side. This was tested by illuminating the foil with an ${ }^{241} \mathrm{Am} \alpha$ source and by recording the scintillation light on the front side with a 9235Q PMT. When the foil was illuminated from the back side, i.e. through the adhesive, almost no additional light was recorded. When the adhesive was removed from the back side the same measurement yielded a small effect. If illuminated on the front side sufficient photons were recorded which could explain the enhancement if applied to the conditions in the water tank. However, this suggests that the $\alpha$ source is either solved in the ultra-pure water or adhering to the front side of the foil. The activity of water from this plant was measured to have an overall $\alpha$ activity in the range of $10^{-6}-10^{-7} \mathrm{~Bq} / \mathrm{kg}$ [23] and measurements of GERDA water samples agree with these values [36]. This activity is too low to explain these excess events. If the front side of the foil had a radon contamination, a higher rate would have been expected after the opening of the water tank. A higher rate was indeed measured but this was in accordance to a higher dark rate after prolonged exposure to light of the Cherenkov PMTs.

Another explanation for the origin of this anomaly are $\beta$ sources since electrons have much higher specific ranges in comparison to $\alpha$ particles. The stainless steel of the GERDA water tank exhibits a low level of radioactivity and one of the strongest contribution to the radionuclides in the steel is the $\beta$ emitter ${ }^{60} \mathrm{Co}$ with an activity of $\sim 20 \mathrm{mBq} / \mathrm{kg}$ [37] 


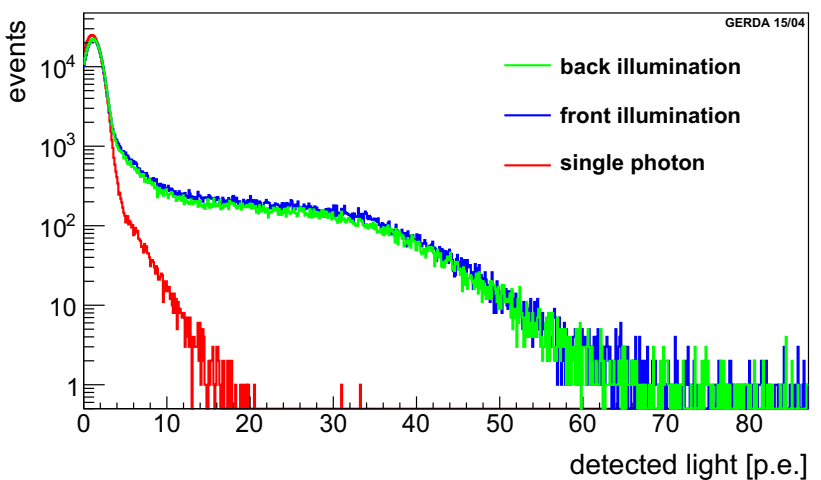

Fig. 14 The effect of irradiation of the VM2000 reflective foil by a $\beta$ source $\left({ }^{60} \mathrm{Co}\right)$ recorded by a PMT. The single photon response $($ red $)$ is compared to an irradiation from the front (blue) or back side (green) which carries the adhesive

which leads to a ${ }^{60} \mathrm{Co}$ activity on the surface of the water tank of $\sim 5 \mathrm{~Bq}$. To test the effect the foil was illuminated by ${ }^{6}{ }^{60}$ Co-source $\left(Q_{\beta}=0.35 \mathrm{MeV}\right)$ and the results are shown in Fig. 14.

The foil was illuminated from the front and from the back side (adhesive not removed). The back illumination shows only a slightly smaller scintillation effect in comparison to the front illumination and in both spectra a low-energy $\beta$ spectrum emerges. In order to determine an efficiency for this process, the scintillation rate of a $5 \mathrm{~mm}$ thick sheet of plastic scintillator was recorded that is assumed to have an efficiency of unity. By comparing the rate of the foil with the rate of the scintillator, the efficiency of the foil toward $0.35 \mathrm{MeV} \beta$ particles can be calculated:

$\varepsilon_{\beta}^{\text {foil }}=\left(12.0_{-1.0}^{+1.1}\right) \%$

With this efficiency, the activity expected from ${ }^{60} \mathrm{Co}$ from the steel is reduced to $\sim 0.6 / \mathrm{s}$ which is still too high in comparison to the measured rate. Using the mean light recorded by the illumination measurements, the solid angle $(8.5 \%$ of $4 \pi)$ and efficiency $(0.25)$ of the PMT used for this test, the efficiency $(0.3)$ and surface coverage $(0.005)$ of the PMTs in the GERDA water tank the effect of this scintillation can be calculated. It is found that the bulk of these events would deposit 2-6 p.e. in the PMTs per event. These events will on average not fulfill the trigger conditions given in Sect. 2.4. It is still likely that about $1 \%$ of these events could still trigger the veto. This would put the expected and measured rate as well as the expected photon yield of one to two p.e. per PMT into the same order of magnitude. Thus, the scintillation caused by $\beta$ particles has to be considered the most likely source of this enhancement.

\section{Summary}

In this work, the muon veto deployed during Phase I of the GERDA experiment was introduced,its hardware was presented and its performance was shown. In addition, the cosmogenic components of the background in GERDA was systematically identified, analyzed, and compared to Monte Carlo simulations.

The hardware thresholds were chosen in a way that by design a very pure muon sample is recorded with only a few percent contamination by random coincidences or other sources of background. With this powerful muon veto over two years of data have been recorded including the 491 days coincident with the germanium detectors.

The Monte Carlo simulations of earlier work were extended to accommodate for a more realistic set-up of the muon veto. PMT multiplicity and p.e. spectra were found in good agreement with the data and the light deposition of cosmogenic and energy-depositing muons could be related to their different track-lengths in the water tank. With the updated geometry a detection efficiency of $\varepsilon_{\mu d}^{\mathrm{MC}}=$ $(99.935 \pm 0.015) \%$ was found for the simulated energydepositing muons in the water tank.

The muon events in the germanium detectors were studied in detail. A cut on the signals of the germanium detectors was defined that identifies muon hits. From comparison to coincident muon events a rejection efficiency of $\varepsilon_{\mu r}=\left(99.2_{-0.4}^{+0.3}\right) \%$ was found. The events would have produced a background index of $\mathrm{BI}_{\mu}=(3.16 \pm 0.85) \times$ $10^{-3} \mathrm{cts} /(\mathrm{keV} \cdot \mathrm{kg} \cdot$ year $)$.

Acknowledgments The work of the Tübingen group within the GERDA experiment is supported by the Grants 05CDVT1/8, 05A08VT1, 05A11VT3 and 05A14VT2 provided by the BMBF/PT-DESY. We express special thanks to the mechanical and electronic workshops in Tübingen for their assistance during the preparation of the Cherenkov veto. M. Uffinger assisted in the activity measurements of the VM2000. The authors would like to thanks B. Schwingenheuer for useful discussions and for carefully reading the manuscript.

Open Access This article is distributed under the terms of the Creative Commons Attribution 4.0 International License (http://creativecomm ons.org/licenses/by/4.0/), which permits unrestricted use, distribution, and reproduction in any medium, provided you give appropriate credit to the original author(s) and the source, provide a link to the Creative Commons license, and indicate if changes were made.

Funded by SCOAP ${ }^{3}$.

\section{References}

1. K.-H. Ackermann et al., GERDA-collaboration, Eur. J. Phys. C 73, $2330(2013)$

2. M. Agostini et al., GERDA-collaboration, Phys. Rev. Lett. 11, 122503 (2013)

3. M. Agostini et al., GeRDA-collaboration, Eur. Phys. J. C 75, 416 (2015) 
4. C. Cattadori et al., GERDA-collaboration, Nucl. Particle Phys. Process. 265-266, 38 (2015)

5. S. Ahlen et al., MACRO-collaboration, Astrophys. J. 412, 301 (1993)

6. E. Gschwendtner et al., Conf. Proc. C130512 (2013) pp.MOPEA058

7. M. Knapp, PhD thesis, University of Tübingen, 2009

8. F. Ritter, $\mathrm{PhD}$ thesis, University of Tübingen, 2012

9. K. Freund, PhD thesis, University of Tübingen, 2014

10. M. Knapp et al., Nucl. Instr. Methods A 609, 280 (2010)

11. F. Ritter et al., Nucl. Instr. Methods A 617, 420 (2010)

12. G. Heusser, Ann. Rev. Nucl. Part. Sci. 45, 543 (1995)

13. $9354 \mathrm{~KB} / 9350 \mathrm{~KB}$ series PMT, ET Enterprises Limited, Uxbridge

14. G. Bellini et al., J. Instr. 6, P05005 (2011)

15. Custom made stainless steel capsule, Raff + Grund GmbH, Freiberg a.N.,

16. 161403 Mineral oil for IR spectroscopy, Sigma-Aldrich, St. Louis

17. UR5041 polyurethane, Electrolube, Hannover

18. SILGEL $612 \mathrm{~A} / \mathrm{B}$, Wacker Chemie AG, München

19. Custom made magnetic shield, ET Enterprises Limited, Uxbridge

20. RG213/U with custom polyurethane cladding, JoWo Systemtechnik GmbH, Delmenhorst

21. SY1527 Universal Multichannel Power Supply System, CAEN S.p.A., Viareggio
22. A1733 12 Channel 3 kV/3 mA, 4 kV/ 2 mA Board, CAEN S.p.A., Viareggio

23. M. Balata et al., Nucl. Instr. Methods A 370, 605-608 (1996)

24. Daylighting Film DF2000MA (VM2000), 3M, St. Paul

25. G. Heusser, M. Laubernstein, H. Neder, Radioact. Environ. 8, 495 (2006)

26. M. Agostini et al., Eur. Phys. J. C 75, 506 (2015)

27. Glass Bubbles S32, 3M, St. Paul

28. UPS-923A plastic scintillator, Institute for Single Crystals, Kharkov

29. S-Type Y11(250)M optical fibres, Kuraray Co., Ltd., Tokyo

30. H6780/H6780-2 PMT, Hamamatsu Photonics K.K., Moscow

31. SIS 3301, 8 Channel 105 MS/s 14-bit ADC/Digitizer, STRUCK Innovative Systeme GmbH, 22399 Hamburg

32. PAS 9817/AO analog output card, Precision Analog Systems Co., Plantation

33. S. Agostinelli et al., GEANT4 Collaboration, Nucl. Instrum. Methods A 506, 250 (2003)

34. M. Boswell et al., IEEE Trans. Nucl. Sci. 58, 1212 (2011)

35. L. Pandola et al., Nucl. Instrum. Methods A 570, 149 (2007)

36. M. Balata, Priv. Commun. (2014)

37. W. Maneschg, Nucl. Instrum. Methods A 593, 448 (2008) 